\title{
Musculoskeletal health in the workplace
}

\section{Joanne O. Crawford ${ }^{\mathrm{a},{ }^{*}}$, Danielle Berkovic ${ }^{\mathrm{b}}$, Jo Erwin ${ }^{\mathrm{c}}$, Sarah M. Copsey ${ }^{\mathrm{d}}$, Alice Davis ${ }^{\mathrm{e}}$, Evanthia Giagloglou ${ }^{\mathrm{e}}$, Amin Yazdani ${ }^{\mathrm{f}}$, Jan Hartvigsen ${ }^{\mathrm{g}}$, Richard Graveling ${ }^{\mathrm{e}}$, Anthony Woolf ${ }^{\mathrm{C}}$}

\author{
a Victoria University of Wellington, New Zealand \\ ${ }^{\mathrm{b}}$ Monash University, Australia \\ ${ }^{\mathrm{c}}$ Bone and Joint Research Group, Royal Cornwall Hospital, UK \\ ${ }^{\mathrm{d}}$ European Agency for Safety and Health at Work, Spain \\ e Institute of Occupational Medicine, UK \\ ${ }^{\mathrm{f}}$ Canadian Institute for Safety, Wellness, and Performance - Conestoga College Institute of Technology and \\ Advanced Learning, Canada \\ ${ }^{\mathrm{g}}$ University of Southern Denmark, Nordic Institute of Chiropractic and Clinical Biomechanics, Denmark
}

\section{Keywords:}

Musculoskeletal

Work

Health

Management

Prevention

\begin{abstract}
A B S T R A C T
Musculoskeletal (MSK) problems remain the most frequent reason why individuals are absent from work, including those with workrelated musculoskeletal disorders (WRMSDs or MSDs) and those with chronic MSK problems. This paper aims to examine changes in work and the workforce since 2000; how work impacts on chronic MSK conditions and how we can help people with these conditions to stay at work. While our knowledge of the causes of WRMSDs has increased since 2000, there has been limited workplace action in reducing exposure to hazards. A life course approach is needed as individuals of all ages are reporting MSK problems. How people work has also changed and informalisation of work contracts has increased with a perceived concurrent reduction in occupational safety and health $(\mathrm{OSH})$ protection. Retaining people at work with MSK problems requires compliance with relevant safety, health and diversity legislation and a risk management approach. Good and open communication within the workplace and identification of other sources of support is also
\end{abstract}

\footnotetext{
* Corresponding author.

E-mail addresses: Joanne.crawford@vuw.ac.nz (J.O. Crawford), danielle.berkovic@monash.edu (D. Berkovic), josephine. erwin@nhs.net (J. Erwin), copsey@osha.europa.eu (S.M. Copsey), Alice.davis@iom-world.org (A. Davis), eva.giagloglou@iomworld.org (E. Giagloglou), Ayazdani@conestogac.on.ca (A. Yazdani), jhartvigsen@health.sdu.dk (J. Hartvigsen), Richard. graveling@iom-world.org (R. Graveling), anthony.woolf@btopenworld.com (A. Woolf).
} 
necessary. Considerations must be made at the individual level (internal motivation), organisational level (a supportive manager) and self-management of symptoms. Simple case examples are provided in the paper of what works in practice as well as a proposed research agenda. Increased awareness at all levels of society of MSK health is essential.

(C) 2020 Elsevier Ltd. All rights reserved.

\section{Introduction}

Musculoskeletal (MSK) problems remain the main reason why individuals are absent from work globally. This includes MSK problems associated with work, where associations have been found between poor workplace ergonomics and physical exposures at work or psychosocial risks at work, and usually referred to as work-related musculoskeletal disorders (WRMSDs or MSDs) and MSK problems related to lifestyle behaviours, disease and injuries outside the workplace that individuals come to work with [1,2]. Across the EU, data from EU-28 identifies that from the labour force survey, selfreporting of MSDs was at 54.2\% in 2007 rising to $60.1 \%$ in 2013; no further data are available at this point [3]. In the US and Canada, MSDs have the most impact on organisations' overall health care costs (53\%), followed by cancer (47\%), diabetes (44\%) and cardiovascular disease/heart disease (32\%) [4]. In the US, MSDs comprise about $40 \%$ of all lost-time workplace injuries [5]. In Canada and Ontario (2018), WRMSDs is the number one type of lost-time work injury, accounting for $40 \%$ and $38 \%$ of all lost-time claims, respectively [6,7]. Each year in Canada, MSDs alone create an economic burden of $\$ 25.6$ billion, of which, $\$ 13.9$ billion is due to productivity losses [8]. According to the Government of Ontario, MSDs cost Ontario workplaces hundreds of millions of dollars due to worker absence and lost productivity. Additional indirect costs, when a worker has suffered from an MSD, include overtime and replacement wages, workstation and equipment modifications, administration costs, training costs for replacement workers and reduced quality of outputs. The Global Burden of Disease study [9] estimated that MSK conditions were the second highest contributor to global disability and that $20 \%-33 \%$ of people across the globe live with a painful MSK condition. In Europe alone, there are an estimated 120 million people with a chronic MSK condition [10].

For the purposes of this chapter, chronic MSK conditions are defined as 'those that last more than 12 weeks, including chronic back pain or chronic upper limb disorders, as well as rheumatic diseases, degenerative conditions such as osteoarthritis or osteoporosis or non-specific pain syndromes categorised as chronic' [11]. Thus, while the term chronic MSK conditions covers a variety of symptoms in the body as well as different disease outcomes, there are also common symptoms, including pain, fatigue, functional limitations and tiredness. Additional health issues, that is, co-morbidities and multimorbidity also often occur in people with MSK conditions, including cardiovascular disease, diabetes and depression [12]. This all impacts on quality of life in individuals as well as in societies where economic consequences could be as high as $2 \%$ of gross domestic product across the EU [13].

Having a chronic MSK condition has a pervasive impact on individuals that include fear and worry, social withdrawal and loss of social roles, family strain, and loss of income [14] and while many people want to continue to work there are a number of barriers and facilitators in making this happen. The aims of this chapter are to:

- Consider what has changed in the workplace and the workforce since 2000 and its impact on MSK conditions;

- To understand how work impacts on chronic MSK conditions and the prevention measures that can be taken in the workplace;

- To gain knowledge on how we can support individuals with MSK conditions to stay at or return to work. 


\section{What has changed in the work and in the workforce since 2000 ?}

The evidence base for MSK conditions

Since the year 2000, there have been several different changes in the world of work which are likely to have had an impact on occurrence of MSK conditions and disability for those working with MSK conditions. This includes an increase in knowledge about the workplace, lifestyle and individual factors that are implicated in the development of MSK conditions including [1,2]:

- Workplace Factors

○ Heavy physical work

- Sedentarism

- Repetitive work

- Poor and/or awkward postures

- Exposure to psychosocial risks

- Lifestyle Factors

- High body mass index

- Smoking

- Physical activity and sport during leisure time

- Individual Factors

- Gender (being female)

$\circ$ Age

- MSK conditions arising not related to work (i.e. rheumatoid arthritis)

- General health and co-morbidity

How the workforce has changed

While these risk factors have been identified, there are also more vulnerable individuals in the workplace. For example, Hanvold et al. [15] identified that $69 \%$ of young adults $(n=420)$ were reporting pain in one of four body regions at the start of a cohort study with only $12 \%$ reporting no pain at baseline. Following up at 20 different times in a 6.5-year period between school, further training and work, the prospective analysis found positive associations between being female and reporting pain in one or more sites $(R R=1.02,95 \% \mathrm{CI} 1.02-1.42)$, mechanical workload $(R R=1.01$, $95 \% \mathrm{CI} 1.00-1.01)$, perceived muscle tension $(\mathrm{RR}=1.02,95 \% \mathrm{CI} 1.01-1.03)$ and tobacco use $(\mathrm{RR}=1.16$, $95 \% \mathrm{CI}, 1.06-1.26$ ); with higher socioeconomic status having a protective effect ( $\mathrm{RR}=0.88,95 \% \mathrm{CI}$ $0.79-0.98)$.

Demographic changes have resulted in more at-risk workers in employment, including women workers and older workers [16]. This emphasises the need to manage workplace hazards for all workers across the life course as we have previously seen younger workers who are also a vulnerable group, are starting work with MSK problems. We need to better understand the health status of individuals at work and this will be discussed in the final section of this chapter.

Finally, there are estimated to be 150 million migrant workers globally. A systematic review by Hargreaves [17] that included 36 papers found that migrant workers are more at risk of an occupational illness or a workplace injury. What has not been assessed is whether support mechanisms are in place to protect migrant workers in relation to occupational safety and health or their training needs.

When we consider that individuals are starting work with MSK problems and are more likely to get problems as they get older, there is a clear need to take a life course approach to prevention and management of MSDs. This is likely to include education of workers as well as workplace interventions to retain individuals at work.

\section{Where people work}

Where people work has also changed since the year 2000, including ongoing urbanisation and industrialisation in low- and middle-income countries, changes in the number of people in different 
sectors, the technology used in the workplace and changes in methods of employment. While there has been an increase in our knowledge levels regarding risk factors for, or exacerbation risks from work; exposure to these risk factors has not reduced. The European Survey of Enterprises on New and Emerging Risks (ESENER 2 and 3) reports, highlight that exposures to specific MSK risks have not reduced across the European Union [18,19]. As Fig. 1 shows, there is increased reporting of the movement of people or heavy loads and exposure to repetitive arm or hand movements.

While there have been traditional sectors, including construction and agriculture, where the risk of damage is higher, people moving into health and social care are still exposed to MSK risks [20]. As work has changed and people have moved into new work sectors, this has not necessarily reduced their exposure to MSK risks. From European sectoral data, in 2017, there were 32.5 million workers employed in manufacturing, this number had reduced by 2 million in the preceding 10 years, whereas the number of workers in public administration, defence, education, human health and social work has increased from 51.4 to 55.5 million in 2017; and those in wholesale and retail trade, transport, accommodation and food services which has increased by 2 million workers: both sectors where high demands are made on the MSK system [20].

\section{How people work}

We are also seeing a change in the way we work. Digitalisation means we can work anywhere and at any time, and the use of less formal contracts within the gig economy does mean we need to question whether health and safety is still covered in these informal jobs. EU OSHA (2017) examined potential occupational safety and health (OSH) risks in a discussion paper on online platform working where individuals are perceived to be self-employed [21]. Furthermore, our retail habits are changing and the use of e-retail to purchase products has increased the numbers of workers in distribution centres and those involved in delivery; who are not always in protective workplaces [22].

The informalisation of sectors through the gig economy or online platform working does bring with it the potential for a reduction in OSH protection where individuals are pseudo self-employed. This has been seen with other groups including part-time, temporary and migrant workers, so there is a clear need to be able to define the role of OSH with these workers in reducing exposure to poor ergonomics [20].

As technology evolves, the landscape of employment is also changing. Organisations have maximised the performance of their overall system by combining robots and co-robots, for repetitive and monotonous assembly steps, and humans, who have the sensorimotor and creative problem-solving skills to succeed where more flexibility is required [23]. However, according to Nedelkoska \& Quintini [24], automation could eliminate $14 \%$ and significantly disrupt up to $32 \%$ of existing jobs across 32 Organisation for Economic Co-operation and Development (OECD) countries. It is hypothesised that there will be an increase in workplace accidents due to unforeseen interactions with robots; optimal workstation design and choice of suitable safety equipment is vital for these manufacturing workplaces [25]. Additionally, appropriate safety standards for occupational safety are absent for direct human-robot interactions in a collaborative workspace [23]. Therefore, it is not only important to ensure workers attain the appropriate skills to interact and engage with machines, but it is also vital that collaborative workspaces are safe for human operators.

\section{Prevention measures that can be taken in the workplace}

Globally, employers face several challenges in developing a sustainable, healthy and productive workforce. Workplace injury rates and their economic and societal costs are excessively high. MSD risk factors are depicted in the human factors and ergonomics (HFE) systems model [26], as factors related to the individual/person, the work task and the work environment domains. Not surprisingly, some of these individual-level outcomes are related to reduced organisational performance, such as product or service quality and work productivity $[27,28]$. It has been estimated that the number of quality deficits and errors in assembly work may increase upwards of ten times when compared with optimal working conditions [29]. Furthermore, a mismatch between the technical system and the human operator, may be an important intermediary between human factors and detrimental system effects [27]. In a metaanalysis, Yung et al. [28] found that up to $42 \%$ of variance in manufacturing quality deficits were 


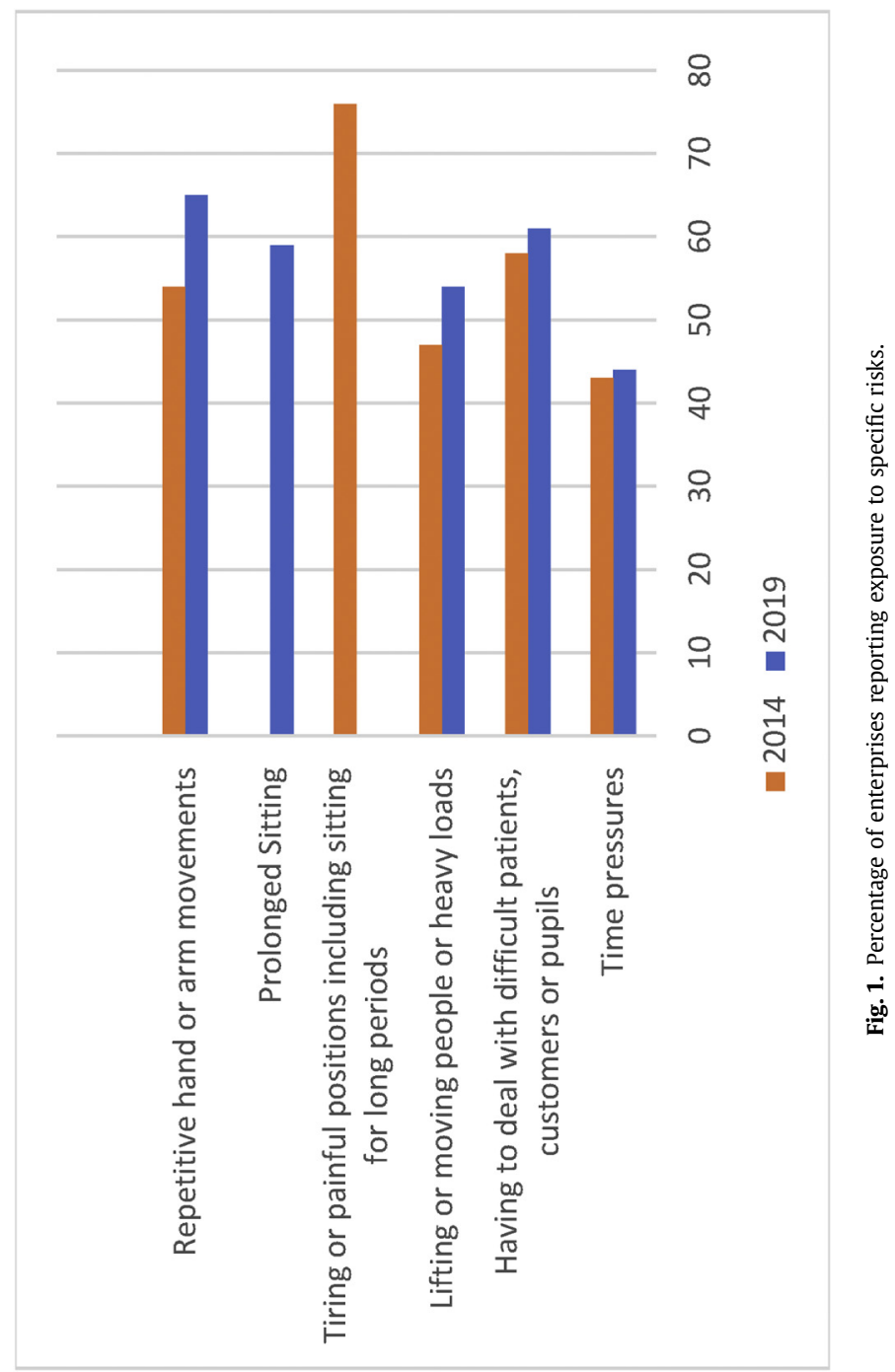


accounted for by operator fatigue. This variance is likely to be dependent on the complexity, precision and forces required to complete the task, as well as environmental workplace factors.

There are some evidence on the success in the effectiveness of MSD prevention activities and ergonomic interventions to reduce the risk of MSDs in the workplace [30-34]. Such interventions consider the interactions among humans and other elements of a system to optimise human wellbeing and overall system performance [35]. In other words, fitting the work to the human. Currently, the hierarchy of hazard controls is a widely accepted system used as standard practice for the prevention of MSD and other adverse outcomes [36]. This consists of selecting controls on the basis of five broad methods: elimination, substitution, engineering controls, administrative controls and personal protective equipment. These control methods are mainly targeted to workers' exposures within individual tasks or actions, solving a specific threat or problem or a set of problems. Unfortunately, the dissemination, adoption and implementation of these control actions are slow and challenging [37]. This is because controls are often implemented as part of a stand-alone ergonomics programme [34], yet the lack of coherency within existing business processes minimises their effectiveness and sustainability. Additionally, an isolated approach is vulnerable to financial downturns [34].

There are several barriers to implement a successful MSD prevention programme in the workplace [38]. The challenges that organisations may face to prevent workplace injuries, including MSDs can be addressed by integrating injury prevention activities and approaches, using harmonised concepts and terminology, into existing broader management systems and frameworks (i.e. quality and environment management systems) [34,38,39]. By integrating prevention activities into central business functions, a novel integrative approach is taken which is sustainable and can be positioned at an organisationallevel using a continuous improvement approach [34,39]. Previous research found that integrating injury prevention into a broader organisational framework and management systems, not only leads to a healthier, safer, and more productive workforce, but also enhances organisational success and overall performance [39].

It is essential that the tools used for hazard identification, risk assessment and risk management are tailored towards organisations that may have limits on time, personnel and financial resources. For instance, small businesses may lack the knowledge of manual material handling and resulting injuries, and often have limited time and budget to address these issues [40]. Hence, it is essential to create costeffective and scalable solutions that fit the complexity of different organisations. Since small businesses make up the majority of businesses and employ most of the workers in the private sector, integrating MSD prevention into a broader management framework and into day-to-day business operations could have a large societal impact. This integration supports the inclusion of ergonomics into the design of the work environment and procurement of equipment, tools, and materials, which may result in significant enhancements in safety and performance [41,42]. Recent attempts in creating a scalable MSD prevention guideline, such as a new MSD Prevention Guideline in Ontario, Canada [43] provides a comprehensive resource to assist organisations with the implementation of MSD prevention programme in their workplace and to improve their organisational performance. This programme has been designed to work in all business sizes. This is an example of a collaborative effort to develop a scalable prevention guideline tailored towards the specific need of micro-small businesses with significant involvement of stakeholders including smat and micro-businesses.

Standards, guidelines and best practices provide a method to introduce terms, concepts, minimum requirements and ergonomic principles to organisations, end-users and designers of products, tools, equipment and services to facilitate the implementation of MSD prevention measures at an organisational level [44]. They can also be utilised as resources to foster innovation of new products and technologies and to increase competitive advantage in the global economy [45,46]. Standards could be referenced in legislation and, therefore, become a significant part of compliance requirements. While there is tremendous value in standards and guidelines as tools to foster innovation and as a mechanism to implement workplace injury prevention measures, there remains trepidation around using standards to their fullest potential. Standards and guidelines may be lacking in the following areas: evidence-based data (due to availability), full transparency (conflict of interest in development), scientifically supported methods (controlling for confounding factors, understanding combined and interactive effects), and sensitivity and specificity of threshold values for numerous potential effects [47]. However, when evidence-informed standards and guidelines are well-designed and 
implemented, their impact has been shown to potentially reduce workplace injuries $[38,48,49]$ and have positive impacts on an organisation's safety, operational and economic performance [50]. For instance, a study by Yung et al. [49] evaluated the impact of the new 2018 American Conference of Governmental Industrial Hygienists (ACGIH) guideline for intensive hand activities in manufacturing; authors found in a large US prospective cohort study that this new guideline, when implemented, can prevent between $11 \%$ and $25 \%$ of cases of Carpal tunnel syndrome in the cohort.

$\mathrm{OSH}$ and prevention activities also need to link to workplace health promotion to promote MSK healthy behaviours. While different risks can be removed or reduced in the work environment [51], there is also a role for health promotion activities in relation to general and MSK health. Individuals can spend more than a third of their time at work so creating health opportunities in the workplace is an important route to improving health [52]. This can start with an increased awareness of the importance MSK health and the impacts of any chronic problems to the general workforce [53]. Specifically, aspects of health behaviours, including improving eating habits, increasing physical activity, avoiding sedentary behaviour and smoking cessation can all benefit the individual with MSK problems.

\section{How to enable people with MSK problems to remain in work}

Having safe and healthy working conditions is a key component of retaining workers with chronic diseases and disabilities and making work sustainable across the life course. While work should not exacerbate existing health conditions, a broader objective is that work should promote health and wellbeing for all workers.

The legislative approach

Modern employment legislation generally requires employers to comply with two sets of complimentary legislative areas, health and safety legislation (invoking the hierarchy of prevention measures) and disability discrimination legislation requiring reasonable adjustments to be made.

\section{Health and safety legislation}

Modern occupational safety and health legislation requires employers to prevent risks, based on risk assessments. The priority is to eliminate risks at source, take collective measures to make work safer and healthier for all workers and adapt work to workers. For example, the European Union Member States [54], Australia [55] and Canada [56], follow the risk assessment approach. It is promoted by the International Labour Organisation (ILO) [57] and covered by an ISO standard [58]. The approach is important, as measures to make work easier for all workers could enable someone with a chronic health condition to continue working. The EU legislative approach, for example, requires particularly sensitive groups, such as workers with chronic conditions, to be protected against hazards that specifically affect them. This is also part of the ILO approach. Making workplaces more inclusive for all workers, for example, when buying equipment, planning tasks or altering buildings, reduces the need to make adjustments for individuals. In some countries, such as in the EU, additional legislation setting out minimum requirements for workplaces requires employers to take account of disabled workers, in particular regarding doors, passageways and staircases, washbasins and lavatories, etc. and workstations used or occupied directly by disabled persons [59].

\section{Employment disability discrimination legislation}

Various countries have in place legislation which requires employers to provide reasonable accommodation or workplace adjustments for employees with disabilities, such as providing equipment, adapting hours of work, changing tasks or providing training [60,61]. This is complementary to health and safety legislation which requires risk assessment, adapting work to workers and taking account of sensitive groups. 
Incorporating diversity into MSD risk assessment

Health and safety risk assessment needs to be sensitive to diversity and individual differences, to effectively deliver both collective measures to reduce risks for all the workforce and individual measures for those at greater risk $[62,63]$. There are a number of success factors for taking account of diversity when assessing workplace health and safety risks and implementing prevention measures, including that:

- Employers must view diversity in the workforce as an asset and be committed to ensuring everyone's health and safety by preventing MSD risks and promoting health and wellbeing, including MSK health.

- Diversity should be taken into account in the design and planning stages, when changes are proposed or new purchases are planned.

- When making the practical assessment it is important to avoid assumptions about who is at risk and how they work and consider everyone, taking account of individual differences.

- It is also important to look at the real work done and how it is carried out in practice.

- Work should be adapted to workers, in general and to take account of differing work abilities where necessary.

- The (consultation) and participation of (disabled) workers and others concerned are key to this. A culture is needed where the workers feel able to disclose health problems and raise health and safety issues that affect them.

- It is important that safety and equalities personnel work together in a company in the areas of both policy and practice.

- Managers and workers, safety and human resources personnel need training to successfully manage health and safety in a manner sensitive to diversity and to understand the needs of different groups, for example, workers with chronic conditions.

- While even small companies will be able to deal with simple situations successfully, expertise should be sought when necessary and especially in more complex situations.

However, the challenge is that many people with an MSK problem do not want to disclose their problems or be labelled disabled. Some are often below the threshold where the label disability can be used. Thus, diversity needs to consider diversity in physical function (or dysfunction), rather than able bodied versus disabled people.

\section{Work ability}

While much is said about health and work, it is important to understand that a person does not need to be $100 \%$ fit to actually come to work and at times coming back to work earlier, or a graduated return to work can be helpful. Workplace accommodations should be determined by focussing on an individual's work ability, in other words, taking a positive approach and focussing on an individual's capabilities and not their disabilities. While the phrase work ability has come to us through assessment of older workers, there is now a diverse age range in the workforce due to the extension of working lives. Some countries, such as Finland and Austria, have developed work ability assessment methods [16,52]. These approaches focussing on work ability have been developed in response to the need to retain ageing workers, by adapting work in general for an ageing workforce and making specific adjustments for individuals where necessary.

\section{Early intervention and early access to advice}

The earlier a problem is reported, the easier it is to deal with; an employer cannot take action if they are not aware of the problem. However, this needs an open communication environment where individuals feel able to report their problems. This involves encouraging and enabling employees to disclose health problems as soon as they arise, assuring them that they will be listened to and supported 
and that they should seek medical advice as soon as possible. Medical advice, if shared with permission, should help the employer to understand what support the worker needs. Often, the necessary accommodations can be identified through discussion between the worker and their manager. For more complex situations, advice can be sought from professionals, such as ergonomists, safety engineers, occupational health professionals, occupational therapists and physiotherapists. In large organisations, it is crucial that occupational health and human resources collaborate on providing support [53]. Communication needs to be consistent across organisations and supervisors need uniform training [64].

\section{Communication and conversations}

Factors at both the individual, interpersonal and organisational levels may influence employee action when experiencing MSK problems. Managers play a key role in relation to workplace health $[65,66]$ and management behaviour influences how an employee handles pain at work [67-69]. Providing possibilities for communication can influence whether an employee can continue working when experiencing pain [70], therefore, the importance of good communication between employers, staff and peers in supporting physical and mental health in the workplace has been recognised by the World Health Organisation (WHO) [71,72]. In the UK, there are numerous resources for line managers providing guidance on how to open up conversations about mental health [73,74] and raise awareness in the workplace, however, there are far fewer resources for MSK health. A recent contribution is the EU OSHA publication 'Conversation starters for workplace discussions about MSDs,' [75] which contains guidance for managers and employees on communicating about MSK conditions. It has been designed for use in facilitating group discussions in the workplace or during training. The conversation starters cover different scenarios, including tackling MSK conditions in a range of different jobs; telling an employer about a diagnosis of a MSK disease; speaking to a worker who may be suffering from back pain and tackling MSK conditions in a workplace where tasks are highly differentiated by gender. This resource makes clear the benefits of having honest and constructive conversations as soon as a problem arises.

An open, positive culture has been shown to be central to establishing a healthy workplace which enables employees to care for their own health, receive early treatment and support, and continue to work even if they have some limitations [76,77]. It enables open conversations and avoids employees feeling isolated and worried about their physical and mental health. Open communication is encouraged in the workplace if employees feel confident they will be listened to and action will be taken. 'Toolbox Talks' are an example of a sector specific strategy to encourage conversations around reducing risks in the working environment. The construction industry encourages employees - at all levels - to come together as they usually do during their breaks and allow some time for health and safety conversations. Concerns can be easily raised and discussed [73].

There is some evidence that communication of information about MSK pain may reduce absenteeism $[78,79]$. A recent scoping review [80] concluded that while some studies suggest that educational resources can positively influence absenteeism and pain-related loss of workability, there is a gap in knowledge regarding the best content and delivery of education of material in the workplace.

\section{Beginning conversations}

Having that first conversation with employees about MSK health may feel daunting to employers or managers. However, employers are often already actively talking about health and safety, and many workplaces may have wellbeing initiatives. If employers are not already talking about prevention and self-care around MSK health through these initiatives, they can begin to use them to include it. Some of these channels may be promoted by employers, other more informal channels can be promoted by staff and Trade Unions. Communication training should aim to form a workforce that openly discusses health risks. Workers need to understand the communication process: Who should they talk to? What happens when a risk or specific MSK issue is reported? Is there an opportunity to give feedback on any action that was taken? This is particularly important for line managers and those in a position of responsibility for others [81]. 
A worker's needs will become clearer through good communication. A conversation between an individual and their manager about a health condition could cover the following [75]:

- the condition;

- the symptoms experienced;

- if the symptoms vary, how they feel on a good or bad day;

- the effects of medication;

- what tasks they find challenging and need help with;

- what support they need or might need to do their job now and, in the future.

Beyond these communication skills, it is important that employers and managers are able to signpost on to appropriate sources of advice and support and are clear what adaptations, adjustments and support can be offered in the workplace, such as task rotation, reduced hours, equipment and support from colleagues. There also needs to be clear policies on how employers and mangers communicate with employees who are absent from work or on a return to work programme to ensure that engagement is appropriate, timely and proportionate.

Steps towards improving communication about MSK health in the workplace include:

- embed MSK health in inductions and training - Ensure staff are given information on how MSK health is managed and what support is available as part of inductions.

- use internal communication channels to encourage openness - Raise awareness through blogs, myth busters, factsheets, tips for managers, useful web links and frequently asked questions. Use posters, notice boards, staff newsletters, magazines, intranet and internet pages to get the messages out.

- encourage MSK health champions - People at all levels talking openly about MSK health sends a clear message that you will get support if you are experiencing an MSK problem.

\section{Getting advice and support}

Advice available from public services and health disability organisations can be helpful in finding solutions. Some countries have return-to-work support schemes and work accident insurance services that offer advice, support with return-to-work plans and grants for workplace adaptations. The worker's healthcare team should give advice on what tasks are appropriate and what should be avoided.

\section{Universal design and inclusive workplaces}

Workplaces designed to take account of all peoples' capabilities and limitations will help prevent work loss by decreasing obstacles and making tasks easier [82]. This can be termed universal design, inclusive design, accessible design and design. Regardless of slight differences in approach, the goal of all is the same [83]. Some examples include:

- adjustable furniture, adjustable seating and desks;

- reducing physical effort and minimising fatigue involved in manual tasks;

- tools with textured grips with a diameter which minimises grasping force;

- automated doors and accessible entrances;

- offering working from home, part-time working and flexi-time and job sharing to all the workforce.

The more workplaces are designed to be inclusive and accessible for all, the easier it will be for workers to continue working with a chronic condition, without the need for additional accommodations. This is not just about planning new buildings. Every time a modification is made to workplace or new equipment purchased it provides an opportunity to improve inclusivity, for example by purchasing more ergonomic tools or height adjustable desks if new desks are to be purchased. 


\section{Summary}

Often health and safety or making work accommodations is erroneously seen as a burden by employers. However, often measures to support workers with health conditions, such as ergonomic furniture and equipment, usually make work easier and the workplace safer and more accessible for all workers and clients as well. Many measures are cheap and simple and designing workplaces to be as accessible as possible for all workers - known as universal design or inclusive design - will benefit everyone.

\section{Facilitators and challenges in enabling worker retention}

MSKs are associated with high rates of worker retention across Europe, North America and Australasia $[10,84,85]$. Individuals with MSK conditions experience reduced productivity [86] and increased absenteeism and presenteeism rates in the workplace. Levels of worker retention vary by job type, for example, people with MSK conditions performing dextrous or laborious manual tasks are at an increased risk of early workforce exit than those in a more inactive and/or flexible environment [87].

For people with MSK conditions, maintaining capacity to work is known to contribute physical benefits, including sustained joint strength, and psychological benefits, such as social engagement and brain activity [88]. In contrast, some workers across the age spectrum with MSK conditions may struggle with the demands of work, and consider a thought-out plan for early retirement as optimal for physical and mental health outcomes [89]. The opposite can also be true: some workers push to maintain their work status to the detriment of their physical capacity, whereas others retire early without proper planning or intervention to attempt to remain at work.

On a global scale, early retirement from MSK conditions is common [90] and this can lead to financial distress and is known to contribute to poorer health outcomes among people with MSK conditions, including severe physical pain, high psychological distress, reduced ability to participate in social roles, and decreased individual quality of life [91].

Lowering rates of MSK-attributable worker retention are not only explained by work demands, but also by individual biomedical (physical traits) and non-biomedical (psychosocial) factors. Work impacts will also fluctuate throughout individual career trajectories. It follows that a combination of medical care and ergonomic modifications and/or improvements, may enable workers with MSK conditions to remain at work where achievable. There is a plethora of evidence surrounding ergonomic modifications and their impacts on worker retention for people with MSK conditions. What is perhaps less known, and imperative for us to understand, are the barriers and enablers in facilitating worker retention. This needs to be person-centred and provide first-person explanations of the enablers and barriers to staying at work; at both the individual and the organisational levels.

The individual

The individual refers to people living with, and working, with MSK conditions. The individual encompasses personal beliefs and perspectives that may enable or prevent productive working. Several studies suggest that internal motivations to work, how people with MSK conditions perceive their capacity to work, and workers' relationship with their physical symptoms comprise the individual barriers and enablers to worker retention.

Internal motivation refers to an individual's personal desire to remain at work. Evidence suggests that for people living with MSK conditions, work can provide purpose, a haven for social support, and financial benefits $[92,93]$. Further, some studies suggest that the motivation to remain at work acts as a distraction from physical symptoms burden and reduces potential psychological burdens of remaining at home with a lack of routine [94]. Qualitative evidence describes people with MSK conditions, specifically inflammatory arthritis types, using the motivation to remain at work to implement ergonomic and/or workplace modifications [95]. Modifications come in different forms, from replacing a standard desk chair to a tailored alternative or altering working hours to a more manageable workload. Despite individual desire to work, it is important to note that some 
individuals have physical symptoms that render them incapable of working within their current field; their internal motivation can determine people's decision to study, or re-train, in a new, MSKfriendly field [96].

In contrast, a major barrier to worker retention is how individuals with MSK conditions perceive their capacity to work. Some with MSK conditions may adopt the mentality that they are less employable than their healthy peers, and that fluctuating MSK symptoms create instability in terms of productivity and fulfilling workplaces roles [97,98]. For men especially, where the stigma of traditional working roles can create additional psychological pressure to remain at work, negative perceptions of their physical capabilities acted as a major barrier to be able to sustain employment $[99,100]$. People with MSK conditions have explained that the chronic nature of living with the condition encompassed constant navigation between managing pain versus managing career expectations, and felt that leaving the workplace was optimal rather than attempting to remain at work [101]. Finally, it has been suggested that some individuals struggle with external perceptions of the limited capabilities of those living with MSK conditions, and adopt the stigmatised sick role as necessary to perform what their family members and friends expect of them, including reduced work hours [102].

\section{The organisation}

The organisation refers to the physical workplace, workplace culture and the role that employers and colleagues play in fostering an inclusive workplace and/or organisational culture for employees with MSK conditions. Managerial and colleague support, or lack thereof, can act as a major enabler and/or barrier to worker retention. Flexible working environments, and environments where employees can be open about their condition, have also been identified as enablers to worker retention.

Individuals with varying MSK conditions have explained that a supportive manager who allows time off for medical appointments, provides a stable working environment in terms of promoting positive workplace culture, and allowances for flexible working arrangements during disease flares, all facilitate worker retention $[103,104]$. It is important to note that flexible working arrangements extend beyond physical intervention; qualitative research suggests that employees with MSK conditions feel appreciative where employers initiate conversation about ways to facilitate worker productivity and retention $[65,105]$.

One of the biggest enablers of employee retention is where managers provide employees with the opportunity for disease disclosure in the workplace. Evidence suggests that those with open chances to talk about their condition and symptoms in the workplace were more capable of staying at work and working productively [106,107]. However, where employees do not conceal their condition, studies note that the invisibility of symptoms can lead other 'healthy' colleagues and managers to doubt the genuineness of symptoms [93]. These doubts and tensions often arise from peers with minimal MSKrelated knowledge, and it is suggested that educating workplaces of the prevalence and nature of MSK conditions may broaden understanding and improve worker retention rates [108].

Within the workplace, a major barrier to worker retention is discord with managers and colleagues $[109,110]$. Research suggests that people working with MSK conditions perceive their colleagues to view them as a liability in the workplace, and believe that their 'healthy' colleagues prefer for 'sick' employees to experience high absenteeism rates, rather than presenting to work [93]. Employees with MSK conditions recognise that their colleagues can view them as burdensome, characterised by extra time needed to complete tasks which then becomes the responsibility of the entire workplace [109].

\section{Self-management}

Self-management refers to the 'individual's ability to manage the symptoms, treatment, physical and psychological consequences and life style changes inherent in living with a chronic condition'. [111]. Selfmanagement strategies for people with long-term conditions include patient education and behaviour modification which are designed to build people's feelings of self-efficacy and ability to set goals and take an active role in their own health outcomes and empowering them to have responsibility over the 
decisions and actions being made about their condition [112]. A Swedish study looking at data from over 44,000 patients with hip or knee osteoarthritis found that a structured education and exercise selfmanagement programme had a significant beneficial effect on quality of life, physical activity, pain, use of medication and sick leave [113]. While this evidence highlights the potential benefits from selfmanagement, there is a lack of research on self-management in the workplace.

Having a chronic MSK problem affects all aspects of peoples' lives including work [114]. Evidence suggests that offering disease self-management interventions to employees can result in substantial benefits to employees and employers [115]. Individuals who are aware of their condition and are able to negotiate necessary workplace adjustments are more likely to have improved health and work outcomes [116,117]. People with arthritis were found to have reduced symptoms and increased psychological wellbeing if one or more workplace adjustments were made to improve the management of their condition [117]. Self-management interventions focussing on work or which are based in the workplace are gaining increasing recognition [118]. Research from the US has shown that despite the widespread availability of the Chronic Disease Self-Management Programme (CDSMP), and its known health-related benefits, programme adoption remains low in workplace settings [119]. Stanford's CDSMP has been shown to be very effective in helping individuals better manage their chronic disease and related complications. It has been adapted to fit the needs of the workplace i.e. cost-effective, not too disruptive of work schedules, and achieve varying work-related outcomes (both individual and organisational) within the constraints of the workplace environment [119]. The Workplace CDMSP (wCDSMP) is being delivered across the US. A trial of this intervention found that relative to usual care, wCDSMP participants reported significantly larger improvements in fatigue, physical activity, soda/sugar beverage consumption and mental work limitations [119]. While over 20\% of participants in the trail of wCDSMP had arthritis or an MSK problem, it was not focussed particularly on MSK conditions. In the UK, the Joint Pain Advisor Programme [120] is a lowcost self-management intervention which has been successfully trialled in the community. It is currently being trialled in the workplace, early indications are that it is acceptable to employers and employees and results in reduction in pain, weight loss and increased physical activity.

Self-management initiatives can provide people living with chronic MSK problems with the skills, knowledge, self-confidence and coping strategies to enable them to continue to engage in employment. However, work has not often considered explicitly as an outcome of self-management programmes and the delivery and outcomes of workplace based self-management programmes for MSK problems requires further research.

Key components of a positive workplace culture for MSK health are increasing peoples' ability to communicate about these problems, and supporting self-help, while encouraging the seeking of professional help where needed and including those healthcare professionals in the conversation when possible.

\section{What works in practice?}

While the evidence base in this area of research is still growing, the following section describes eight case studies and the workplace interventions that enabled retention of the worker. The aim of the cases was to examine the journey to either return-to-work or stay at work with a chronic MSD [121].

Interviews were carried out with the eight individuals who had the following MSK health issues:

\begin{tabular}{ll}
\hline Retail worker & Chondromalacia \\
ICT worker & Knee osteoarthritis \\
University lecturer & Osteoporosis \\
Researcher & Upper limb problems \\
Receptionist & Bone fractures due to osteopenia \\
Podiatrist & Neck disc herniation \\
Project manager & Piriformis muscle syndrome and osteopenia \\
Police officer & Rib costochondritis, sciatica, finger pain \\
\hline
\end{tabular}


The majority of participants were female (six out of eight) and worked in sedentary or static roles. All the participants had access to return-to-work programmes but the majority did not take sick leave.

The approach taken to analyse the cases was based on the Biopsychosocial Model [122] to examine the physical, psychological and social factors, influencing the retention at work. The main findings are described below.

Physical workplace changes - tasks that require bodily force or new equipment/tools

- Removal or help with lifting and handling

- Passing tasks onto others such as data entry work

- The provision of stand/sit desks to allow posture change

- Having access to a rest room to carry out stretches

- Technological interventions, adapted technology, voice recognition software

- Testing interventions and taking a trial/error approach

- Risk assessing interventions to ensure no new risks introduced

- Use of teleworking or not travelling during rush hour periods

- Careful planning and booking of international travel

\section{Social/organisational changes}

- Changing working hours to fit around appointments

- Ensuring the employer understands the restrictions on the worker and has the conversation

- Phased return to work worked out with line manager and other stakeholders

- A multidisciplinary approach including health professionals, occupational health professionals, ergonomists, human resources, line managers and the individual

\section{Psychological/individual changes}

- Line manager support

- Support from colleagues

- Open communication - while the worker is on leave and during the return phase

- The individual having control over the return-to-work process

\section{Summary}

These cases have emphasised the importance of good communication, flexibility and having an open culture within the workplaces studied and this was in combination with new (simple or sophisticated) technology can leading to successful retention. Furthermore, simple workplace accommodations were implemented that enabled the worker to stay productive and at work.

\section{Funding statement}

Part of the paper was funded by EU OSHA and this is coming out as a separate report from them. No other funding has been received. 


\section{Research agenda}

MSK conditions are leading causes of work disability everywhere, yet they do not feature prominently on national health policy or research agendas. A review of system policies for prevention and management of non-communicable diseases among OECD countries found that in spite of the large societal burden MSK conditions did not feature prominently [123], and a recent study from Denmark showed that in spite of back and neck pain being the most burdensome conditions in terms of years lived with disability, only $0.88 \%$ of national health research budgets were spent on identifying effective prevention and treatment strategies [124] - and that included research related to the workplace. Thus, the first call to action in a research agenda regarding MSK conditions everywhere is to call on governments, NGOs, companies, foundations and everyone else involved in research funding to prioritise funding of research related to all aspects of MSK conditions and in particular as they relate to the workplace and to work disability. This is crucially important because it is through work, we mainly gain social contact, income, stability, purpose and opportunities to demonstrate our skills and achievements.

A research agenda in work and MSK conditions should therefore include:

1. Generation of new knowledge that leads to improved health for workers everywhere

- Research into evidence-based programmes for young people in order to prepare them for the mental and physical stressors they will encounter in the workplace as well as evidencebased programmes for integration of young people who already have MSK conditions into workplaces [125].

- Research into evidence-based programmes aiming to overcome physical, mental and social barriers for people with persistent MSK conditions so they are supported in remaining in the workplace

- Research to provide simple tools for workplaces to assist evaluations of adverse workplace exposures and determine suitable adjustments

- Determine the drivers for work disability at the personal, workplace and societal level in order to identify levers for achieving effective prevention

- Conduct high-quality clinical trials to demonstrate effectiveness of workplace interventions in order to inform health and work policy decisions

2. Research into better integration of healthcare and workplace interventions directed towards workers with work disability

- Research into how misconceptions about MSK conditions and work among health professionals, workers, workplaces and the media can be addressed effectively

- Research that identifies barriers and facilitators for integration of healthcare and workplace interventions at the practitioner, workplace and societal level

- Development of models of communication and integration of healthcare and workplace interventions for workers with MSK conditions

- Development of a common language between health professionals and workplaces aimed at emphasising health and participation rather than disease and disability

- Research into how remaining at work or returning to work can be implemented in clinical practice as a valued clinical outcome

- Research into effectiveness and cost-effectiveness of integration of healthcare and workplace interventions

3. Research into how knowledge can be implemented in health policy, care pathways and compensation systems in order to effectively prevent work disability

- Invest in implementation research to address evidence-practice gaps across healthcare and workplaces

4. Research into how knowledge and best practice can be implemented in the workplace

- Research in better understanding MSD aetiology, hazard identification, risk assessment, and identifying effective interventions.

- Research to inform ergonomic design of workplaces and products

- Research in addressing behaviour change in the workplace by employers and employees

- Use a field-to-lab-to-field approach to experimentally design innovative solutions and concepts using realistic exposures, demands, work environments and real users. 
Synthesise existing evidence and use field-to-lab-to-field approach to create evidence and consensus-based standards and guidelines as a systematic implementation strategy to integrate MSD prevention research into organisational management systems.

\section{Practice points}

- Musculoskeletal disorders (MSDs) remain the most frequent reason why individuals are absent from work including those with work-related MSDs and chronic MSDs.

- Our knowledge of workplace factors has increased but there has been limited workplace action to reduce exposure to hazards

- To retain individuals with chronic musculoskeletal (MSK) problems at work, there is a need for a risk management approach encompassing both health and safety legislation, disability legislation and diversity legislation.

- Workplace changes need to be considered at the organisational an individual level, but such changes can often be simple adjustments.

- Having open communication within the workplace can help individuals with chronic health problems to be prepared to discuss those problems and work on solutions.

- There needs to be greater awareness by society, including employers, employees, clinicians and policy makers to value the importance of maintaining MSK health for an active and productive life.

\section{Summary}

While the workplace and workforce have changed since 2000, these changes highlight that exposure to risks for MSDs do not appear to be well managed. Combined with increasing numbers of vulnerable workers including young workers, older workers, women workers and migrant workers and people with other MSK conditions, work disability caused by MSK problems may increase in coming decades. Current recommendations in retaining workers with chronic MSK conditions is to ensure that safety and health regulations are complied with, as well as disability regulations. While there are physical interventions that can be made to help individuals with chronic MSK conditions, other factors, such as including line managers and colleagues in freely discussing MSK conditions and their impacts, better integration of healthcare and the workplace, and social systems that support remaining at work despite some level of pain and disability are also important. Above all, an intensified research effort into developing and implementing effective preventive strategies, including promoting MSK health as well as models of employment that enables workers with MSK conditions to remain at work are necessary to ensure the future wellbeing of individuals and prosperity of societies. For this to happen their needs to be a greater awareness by society, which includes employees, the public, employers, clinicians and policy makers, to value the importance of maintaining MSK health for an active and productive life both in the workplace and outside the workplace.

\section{Declaration of competing interest}

No other conflicts of interest were reported.

\section{References}

[1] da Costa BR, Vieira ER. Risk factors for work-related musculoskeletal disorders: a systematic review of recent longitudinal studies. Am J Ind Med 2010;53(3):285-323. 
[2] Coggon D, Ntani G, Palmer KT, et al. Disabling musculoskeletal pain in working populations: is it the job, the person, or the culture? Pain ${ }^{2}$ 2013;154(6):856-63.

[3] EUROSTAT. Persons reporting a work-related health problem by sex, age and educational attainment level. Available at: https://appsso.eurostat.ec.europa.eu/nui/show.do?dataset=hsw_pb1lang=en. [Accessed 4 March 2020].

[4] International Foundation of Employee Benefit Plans. Workplace wellness trends 2019 survey report. International Foundation of Employee Benefit Plans; 2019.

[5] US Department of Labour. Bureau of Labor Statistics; 2016. . [Accessed 5 March 2020].

[6] Association of Workers' Compensation Boards of Canada. 2018 Lost time claims in Canada. Available at: http://awcbc.org/ ?pageid $=14 ; 2018$

[7] Workplace Safety and Insurance Board. Report builder by the numbers. Available at: http://www.divxy123.ca/ ReportBuilder2017/Pages/report_builder.php; 2018.

[8] Workplace Safety and Prevention Services. Ergonomics. Available at:. 2020. https://www.wsps.ca/Information-Resources/ Topics/MSDs.aspx. [Accessed February 2020].

[9] James SL, Abate D, Abate KH, et al. Global, regional and national incidence, prevalence, and years lived with disability for 354 diseases and injuries for 195 countries and territories, 1990-2017: a systematic analysis for the Global Burden of Disease Study. Lancet 2018;392(10159):1789-858.

[10] EULAR. Horizon 2020 Framework ProgrammeEULAR's position and recommendations. European Agency Against Rheumatism; 2011.

[11] EU OSHA. Workplace health promotion for employees - fact sheet 94. 2010.

[12] Hartvigsen J, Natvig B, Ferreira M. Is it all about a pain in the back? Best Pract Res Clin Rheumatol 2013;27(5):613-23.

[13] Bevan S. Economic impact of musculoskeletal disorders (MSDs) on work in Europe. Best Pract Res Clin Rheumatol 2015; 29(3):356-73.

[14] MacNeela P, Doyle C, O'Gorman D, et al. Experiences of chronic low back pain: a meta-ethnography of qualitative research. Health Psychol Rev 2015;9(1):63-82.

[15] Hanvold TN, Lunde L, Koch M, et al. Multisite musculoskeletal pain among young technical school students entering working life. BMC Muscoskel Disord 2016;17(1):82.

[16] EU OSHA. The ageing workforce: implications for occupational safety and health: a research review. 2016.

[17] Hargreaves S, Rustage K, Nellums LB, et al. Occupational health outcomes among international migrant workers: a systematic review and meta-analysis. Lancet Global Health 2019;7(7):e872-82.

[18] EU OSHA. Enterprises on new and emerging risks (ESENER-2) overview report: managing safety and health at work. EU OSHA; 2016.

[19] EU OSHA. Third European survey of enterprises on new and emerging risks (ESENER 3). EU OSHA; 2019.

[20] (in press) EU OSHA. MSDs Prevention: an exploratory review. 2020.

[21] EU OSHA. Protecting workers in the online platform economy: an overview of regulatory and policy developments in the EU. An overview of regulatory and policy developments in the EU a discussion paper. EU OSHA; 2017.

[22] EU OSHA. The future of the (E-) retail sector from an occupational safety and health point of view, a discussion paper. EU OSHA; 2018.

[23] Faber M, Bützler J, Schlick CM. Human-robot cooperation in future production systems: analysis of requirements for designing an ergonomic work system. Procedia Manuf 2015;3:510-7.

[24] Nedelkoska L, Quintini G. Automation, skills use and training. 2018.

[25] Helander MG. Ergonomics and safety considerations in the design of robotics workplaces: a review and some priorities for research. Int J Ind Ergon 1990;6(2):127-49.

[26] International Organization for Standardization. Ergonomics general approach, principles and concept. 2011. ISO 26800: 2011). ISO 2016.

[27] Kolus A, Wells R, Neumann P. Production quality and human factors engineering: a systematic review and theoretical framework. Appl Ergon 2018;73:55-89.

[28] Yung M, Kolus A, Wells R, et al. Examining the fatigue-quality relationship in manufacturing. Appl Ergon 2020;82: 102919.

[29] Eklund JA. Ergonomics and quality management-humans in interaction with technology, work environment, and organization. Int J Occup Saf Ergon 1999;5(2):143-60.

[30] Tompa E, Dolinschi R, Natale J. Economic evaluation of a participatory ergonomics intervention in a textile plant. Appl Ergon 2013;44(3):480-7.

[31] Integration of ergonomics into PVA-Kaizen interventions: a feasibility study. In: Proceedings of the 16th world congress of the International Ergonomics Association; 2006.

[32] Monroe K, Fick F, Joshi M. Successful integration of ergonomics into continuous improvement initiatives. Work 2012; 41(Supplement 1):1622-4.

[33] Bolis I, Sznelwar LI. A case study of the implementation of an ergonomics improvement committee in a Brazilian hospital-Challenges and benefits. Appl Ergon 2016;53:181-9.

[34] Yazdani A, Neumann WP, Imbeau D, et al. Prevention of musculoskeletal disorders within management systems: a scoping review of practices, approaches, and techniques. Appl Ergon 2015;51:255-62.

[35] International Ergonomics Association. Definition and domains of ergonomics. Available at: https://www.iea.cc/whats/. Accessed 05/03/, 2020.

[36] National Institute for Occupational Safety and Health. Hierarchy of controls. Available at: https://www.cdc.gov/niosh/ topics/hierarchy/default.html. Accessed 05/03/, 2020.

[37] NORA Musculoskeletal Health Cross-Sector Counci. National occupational research agenda for musculoskeletal health. NORA; 2018.

[38] Yazdani A, Wells R. Barriers for implementation of successful change to prevent musculoskeletal disorders and how to systematically address them. Appl Ergon 2018;73:122-40.

[39] Yazdani A, Neumann WP, Imbeau D, et al. How compatible are participatory ergonomics programs with occupational health and safety management systems? Scand J Work Environ Health 2015:111-23. 
[40] MacEachen E, Kosny A, Scott-Dixon K, et al. Workplace health understandings and processes in small businesses: a systematic review of the qualitative literature. J Occup Rehabil 2010;20(2):180-98.

[41] Pinder A. Literature review: barriers to the application of Ergonomics. Human Factors in engineering design. United Kingdom: Health and Safety Executive; 2015.

[42] Du B, Boileu M, Wierts K, et al. Exploring the need for and application of human factors and ergonomics in ambulance design standards: overcoming the barriers (Under Review). Appl Ergon 2020.

[43] Centre of Research Expertise for the Prevention of Musculoskeletal Disorders. MSDs preventiion. Available at:. 2019. www.msdprevention.com. [Accessed 5 March 2020].

[44] Nachreiner F. Standards for ergonomics principles relating to the design of work systems and to mental workload. Appl Ergon 1995;26(4):259-63.

[45] Smart Z. Paving the way for innovation. International Electrotechnical Commission E-tech News and Views; 2018.

[46] BSI Group. Encouraging innovation and growth with standards. Available at:. 2020. https://www.bsigroup.com/en-CA/ Standards/Benefits-of-using-standards/Encouraging-innovation-and-growth-with-standards/. [Accessed 5 March 2020].

[47] Armstrong TJ, Burdorf A, Descatha A, et al. Scientific basis of ISO standards on biomechanical risk factors. Scand J Work Environ Health 2018;44(3):323-9.

[48] Du B, Boileau M, Wierts K, et al. Existing science on human factors and ergonomics in the design of ambulances and EMS equipment. Prehosp Emerg Care 2019;23(5):631-46.

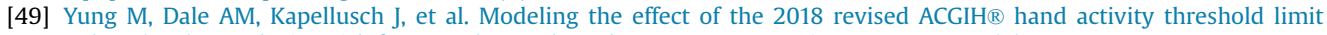
Value $\left.{ }^{(T L V}\right)$ at reducing risk for Carpal tunnel syndrome. J Occup Environ Hyg 2019;16(9):628-33.

[50] Lafuente E, Abad J. Analysis of the relationship between the adoption of the OHSAS 18001 and business performance in different organizational contexts. Saf Sci 2018;103:12-22.

[51] Graveling Richard. Managing low back conditions and low back pain. Available at: https://oshwiki.eu/wiki/Managing low_back_conditions_and_low_back_pain. [Accessed 13 March 2020].

[52] Morschhäuser M, Sochert R. Healthy work in an ageing Europe. Essen: Federal Association of Company Insurance Funds; 2006.

[53] Woolf A. Working with rheumatic and musculoskeletal diseases. RMDs); 2019.

[54] Council Directive. Directive 89/391/EEC (1989) OSH "Framework Directive"on the introduction of measures to encourage improvements in the safety and health of workers at work. Off J Eur Communites 1989;(No L/183/):1-8.

[55] Safe Work Australia. Model work health and Safety Regulations. Safe Work Australia; 2019.

[56] Canadian Centre for Occupational Health and Safety. Risk assessment: OSH answers. Available at:. 2020. https://www. ccohs.ca/oshanswers/hsprograms/risk_assessment.html. [Accessed 24 February 2020].

[57] International Labour Organisation. Guidelines on occupational safety and health management systems ILO-OSH 2001. ILO; 2009.

[58] International Organization for Standardization. ISO 45001:2018(en) Occupational health and safety management systems - Requirements with guidance for use. Available at:. 2018. https://www.iso.org/obp/ui/es/\#iso:std:iso:45001:ed-1: v1:en. [Accessed 24 February 2020].

[59] Directive C. 89/654/EEC (1989) Concerning the minimum safety and health requirements for the workplace (First individual directive within the meaning of Article 16 (1) of Directive 89/191/EEC). Off J Eur Communities, No L/393/1-12, Brussels 1989.

[60] Australian Human Rights Commission. Reasonable adjustment. Available at: https://www.humanrights.gov.au/about/ news/speeches/reasonable-adjustment. [Accessed 24 February 2020].

[61] Council Directive. Council Directive 2000/78/EC - equal treatment. 2000.

[62] EU OSHA. Workforce diversity and risk assessment: ensuring everyone is covered. EU OSHA; 2009.

[63] EU OSHA, European Agency for Safety and Health. Factsheet 87 - workforce diversity and risk assessment: ensuring everyone is covered Summary of an Agency report. 2009.

[64] Jetha A, Yanar B, Lay AM, et al. Work disability management communication bottlenecks within large and complex public service organizations: a sociotechnical systems study. J Occup Rehabil 2019;29(4):754-63.

[65] Larsen AK, Falkenstrøm S, Jørgensen MB, et al. The role of managers in addressing employees with musculoskeletal pain: a mixed methods study. Int Arch Occup Environ Health 2018;91(3):361-72.

[66] National Institute of Health and Care Excellence. Healthy workplaces: improving employee mental and physical health and wellbeing. Quality Standard (QS147). NICE; 2017.

[67] Dellve L, Skagert K, Vilhelmsson R. Leadership in workplace health promotion projects: 1-and 2-year effects on long-term work attendance. Eur J Publ Health 2007;17(5):471-6.

[68] Sterud T, Johannessen HA, Tynes T. Work-related psychosocial and mechanical risk factors for neck/shoulder pain: a 3year follow-up study of the general working population in Norway. Int Arch Occup Environ Health 2014;87(5):471-81.

[69] Wynne-Jones G, Buck R, Porteous C, et al. What happens to work if you're unwell? Beliefs and attitudes of managers and employees with musculoskeletal pain in a public sector setting. J Occup Rehabil 2011;21(1):31-42.

[70] Linton SJ, Boersma K, Traczyk M, et al. Early workplace communication and problem solving to prevent back disability: results of a randomized controlled trial among high-risk workers and their supervisors. J Occup Rehabil 2016;26(2): $150-9$.

[71] Harnois G, Gabriel P, World Health Organization. Mental health and work: impact, issues and good practices. World Health Organization; 2000.

[72] Burton J, World Health Organization. WHO Healthy workplace framework and model: background and supporting literature and practices. World Health Organization; 2010.

[73] HSE. Talking toolkit: preventing work-related stress. HSE; 2019.

[74] Mental Health First Aid Training. MHFALine managers' resource. MHFA; 2016.

[75] EU OSHA. Conversation starters for workplace discussions about musculoskeletal disorders - an EU OSHA resource for workplaces. EU OSHa; 2019.

[76] Schulte PA, Grosch J, Scholl JC, et al. Framework for considering productive aging and work. J Occup Environ Med 2018 60(5):440. 
[77] Black C. Working for a healthier tomorrow. Working for a healthier tomorrow. London: The Stationery Office; 2008.2008.

[78] Ree E, Lie SA, Eriksen HR, et al. Reduction in sick leave by a workplace educational low back pain intervention: a cluster randomized controlled trial. Scand J Publ Health 2016;44(6):571-9.

[79] Frederiksen P, Indahl A, Andersen LL, et al. Can group-based reassuring information alter low back pain behavior? A cluster-randomized controlled trial. PloS One 2017;12(3).

[80] Palsson TS, Boudreau S, Høgh M, et al. Education as a strategy for managing occupational-related musculoskeletal pain: a scoping review. BMJ Open 2020;10(2).

[81] IOSH. Getting the message? Guidance on communication. 2015.

[82] EU OSHA. Working with chronic musculoskeletal disorders. 2020 (in press).

[83] Persson H, Åhman H, Yngling AA, et al. Universal design, inclusive design, accessible design, design for all: different concepts-one goal? On the concept of accessibility-historical, methodological and philosophical aspects. Univers Access Inf Soc 2015;14(4):505-26.

[84] Australian Institute of Health and Welfare. Chronic musculoskeletal disease. Australian Institute of Health and Welfare; 2019.

[85] Bone and Joint Initiative, USA. The impact of musculoskeletal disorders on Americans - opportunities for action. third ed. Bone Joint Initiative; 2016.

[86] Briggs AM, Woolf AD, Dreinhöfer K, et al. Reducing the global burden of musculoskeletal conditions. Bull World Health Organ 2018;96(5):366.

[87] Yucesoy B, Charles LE, Baker B, et al. Occupational and genetic risk factors for osteoarthritis: a review. Work 2015;50(2): $261-73$.

[88] Palmer KT, Goodson N. Ageing, musculoskeletal health and work. Best Pract Res Clin Rheumatol 2015;29(3):391-404.

[89] Gignac MA, Smith PM, Ibrahim S, et al. Retirement expectations of older workers with arthritis and diabetes compared with those of workers with No chronic diseases. Can J Aging La Revue canadienne du vieillissement 2019;38(3):296-314.

[90] Westhoff G, Buttgereit F, Gromnica-Ihle E, et al. Morning stiffness and its influence on early retirement in patients with recent onset rheumatoid arthritis. Rheumatology 2008;47(7):980-4.

[91] Jeon Y, Essue B, Jan S, et al. Economic hardship associated with managing chronic illness: a qualitative inquiry. BMC Health Serv Res 2009;9(1):182.

[92] Brooks J, McCluskey S, King N, et al. Illness perceptions in the context of differing work participation outcomes: exploring the influence of significant others in persistent back pain. BMC Muscoskel Disord 2013;14(1):48.

[93] Holland P, Collins AM. "Whenever I can I push myself to go to work": a qualitative study of experiences of sickness presenteeism among workers with rheumatoid arthritis. Disabil Rehabil 2018;40(4):404-13.

[94] Howard KJ, Mayer TG, Gatchel RJ. Effects of presenteeism in chronic occupational musculoskeletal disorders: stay at work is validated. J Occup Environ Med 2009;51(6):724-31.

[95] Hanson H, Hart RI, Thompson B, et al. Experiences of employment among young people with juvenile idiopathic arthritis: a qualitative study. Disabil Rehabil 2018;40(16):1921-8.

[96] Gross DP, Park J, Rayani F, et al. Motivational interviewing improves sustainable return to work in injured workers after rehabilitation: a cluster randomized controlled trial. Arch Phys Med Rehabil 2017;98(12):2355-63.

[97] Kalsi P, Turkistani W, Sykes C, et al. "Work is a beautiful thing." Exploring attitudes towards employment in chronic pain (CP) patients attending a pain management programme (PMP). J Vocat Rehabil 2016;44(1):97-107.

[98] Patel S, Greasley K, Watson PJ. Barriers to rehabilitation and return to work for unemployed chronic pain patients: a qualitative study. Eur J Pain 2007;11(8):831-40.

[99] Österholm JH, Björk M, Håkansson C. Factors of importance for maintaining work as perceived by men with arthritis. Work 2013;45(4):439-48.

[100] Pendeke TF, Williamson IR. Half the man I was": exploring accounts of emasculation and estrangement amongst British men living with systemic lupus erythematosus. 2016.

[101] Ryan CG, Lauchlan D, Rooney L, et al. Returning to work after long term sickness absence due to low back pain-the struggle within: a qualitative study of the patient's experience. Work 2014;49(3):433-44.

[102] McCluskey S, Brooks J, King N, et al. Are the treatment expectations of'significant others' psychosocial obstacles to work participation for those with persistent low back pain? Work 2014;48(3):391-8.

[103] Berkovic D, Ayton D, Briggs AM, et al. "I would be more of a liability than an asset": navigating the workplace as a younger person with arthritis. J Occup Rehabil 2019:1-10.

[104] Lempp H, Scott D, Kingsley G. The personal impact of rheumatoid arthritis on patients' identity: a qualitative study. Chron Illness 2006;2(2):109-20.

[105] Haafkens JA, Kopnina H, Meerman MG, et al. Facilitating job retention for chronically ill employees: perspectives of line managers and human resource managers. BMC Health Serv Res 2011;11(1):104.

[106] Gignac MA, Cao X. "Should I tell my employer and coworkers I have arthritis?" A longitudinal examination of selfdisclosure in the work place. Arthritis Care Res 2009;61(12):1753-61.

[107] Helme C, Hegarty RS, Stebbings S, et al. "I actually just really need to stop work sometimes": exploring fatigue-related barriers to employment among people with rheumatic diseases. Muscoskel Care 2019;17(1):97-104.

[108] Holloway I, Sofaer-Bennett B, Walker J. The stigmatisation of people with chronic back pain. Disabil Rehabil 2007;29(18): 1456-64.

[109] Bagcivan G, Cinar FI, Cinar M, et al. Living with pain in ankylosing spondylitis: a qualitative study. Contemp Nurse 2015; 51(2-3):135-47.

[110] Crooks VA. Women's experiences of developing musculoskeletal diseases: employment challenges and policy recommendations. Disabil Rehabil 2007;29(14):1107-16.

[111] Barlow J, Wright C, Sheasby J, et al. Self-management approaches for people with chronic conditions: a review. Patient Educ Counsel 2002;48(2):177-87.

[112] Newman S, Steed L, Mulligan K. Self-management interventions for chronic illness. Lancet 2004;364(9444):1523-37.

[113] Jönsson T, Eek F, Dell'Isola A, et al. The better management of patients with osteoarthritis program: outcomes after evidence-based education and exercise delivered nationwide in Sweden. PloS One 2019;14(9). 
[114] Munir F, Khan HT, Yarker J, et al. Self-management of health-behaviors among older and younger workers with chronic illness. Patient Educ Counsel 2009;77(1):109-15.

[115] Caloyeras JP, Liu H, Exum E, et al. Managing manifest diseases, but not health risks, saved PepsiCo money over seven years. Health Aff 2014;33(1):124-31.

[116] Gignac MA, Badley EM, Lacaille D, et al. Managing arthritis and employment: making arthritis-related work changes as a means of adaptation. Arthritis Care Res 2004;51(6):909-16.

[117] Gignac MA. Arthritis and employment: an examination of behavioral coping efforts to manage workplace activity limitations. Arthritis Care Res: Off J Am Coll Rheumatol 2005;53(3):328-36.

[118] de Silva D. Evidence: helping people help themselves. The Health Foundation; 2011.

[119] Smith ML, Wilson MG, DeJoy DM, et al. Chronic Disease Self-Management Program in the workplace: opportunities for health improvement. Front Public Health 2015;2:179.

[120] MSK Knowledge Hub. Joint pain advisor: a new model of care for managing chronic knee, hip or back pain. Available at:. 2018. https://mskhub.org.uk/joint-pain-advisor-a-new-model-of-care-for-managing-chronic-knee-hip-or-back-pain/. [Accessed 26 February 2020].

[121] (In Press) EU OSHA. Case examples and analysis of working with chronic musculoskeletal disorders. 2020.

[122] Engel GL. The need for a new medical model: a challenge for biomedicine. Science 1977;196(4286):129-36.

[123] Briggs AM, Persaud JG, Deverell ML, et al. Integrated prevention and management of non-communicable diseases, including musculoskeletal health: a systematic policy analysis among OECD countries. BMJ Global Health 2019;4(5): e001806.

[124] Poulsen AG. Obstacle race for researchers: first challenge - to raise money (In Danish). J Dan Med Assoc 2020;182: 99-101.

[125] Harris M, Troelstra SA, Straker L, et al. Multimorbidity is common among young workers and related to increased work absenteeism and presenteeism: results from the population-based Raine Study cohort. Scand J Work Environ Health 2018;44(5):521-9. 